\title{
Comparison of higher-power and conventional power ablation of atrial fibrillation using contact-force sensing catheters: A systematic review and meta-analysis
}

\author{
luqian cui ${ }^{1}$, yingjie $\mathrm{chu}^{2}$, yongmei han ${ }^{1}$, and shujuan dong ${ }^{3}$ \\ ${ }^{1}$ Zhengzhou University People's Hospital \\ ${ }^{2}$ Henan Provincial People's Hospital \\ ${ }^{3}$ Henan Province People's Hospital
}

August 17, 2020

\begin{abstract}
Abstract Background: Contact-force sensing catheters are widely used in catheter ablation. The technique of high-power ablation has gained a growing attention in recent years. Our purpose of this meta-analysis is to compare the efficacy and safety between higher-power and conventional power ablation of atrial fibrillation (AF) by contact-force sensing catheters. Methods: We identified studies through searching MEDLINE, EMBASE, the Web of Science, Scopus and the Cochrane Library from inception up until July 2020. The primary outcomes were the recurrence of atrial tachyarrhythmia and complications. The secondary outcomes were acute reconnections of pulmonary veins(PVs), ablation time, and the total procedural time. Results: We identified four nonrandomized, observational studies (nROS) involving 231 patients with high-power ablation and 239 patients with conventional power ablation. There were insignificant differences in the recurrence rate of atrial tachyarrhythmia ( $14.2 \%$ versus $20.5 \%$, OR: $0.64,95 \% \mathrm{CI}: 0.39$ to $1.04, \mathrm{Z}=1.82, \mathrm{P}=0.07)$ and clinical complications $(1.7 \%$ versus $2.5 \%$, OR: $0.72,95 \%$ CI: 0.21 to $2.47, \mathrm{Z}=0.51, \mathrm{P}=0.61)$ between high-power versus conventional power ablation. The high-power group was fewer in acute $\mathrm{PVs}$ reconnections $(\mathrm{P}=0.0001)$, shorter in ablation time $(\mathrm{P}<0.0001)$, and the total procedural time $(\mathrm{P}$ $<0.0001)$ compared with conventional power group. Conclusion: High-power ablation of AF was safe and efficient compared with that of conventional power ablation, and reduced ablation time and the total procedural time.
\end{abstract}

\section{Hosted file}

HPSD.docx available at https://authorea.com/users/351511/articles/476175-comparison-ofhigher-power-and-conventional-power-ablation-of-atrial-fibrillation-using-contact-forcesensing-catheters-a-systematic-review-and-meta-analysis

\section{Hosted file}

Table1.docx available at https://authorea.com/users/351511/articles/476175-comparison-ofhigher-power-and-conventional-power-ablation-of-atrial-fibrillation-using-contact-forcesensing-catheters-a-systematic-review-and-meta-analysis 
Figure1. Flow diagram of the study selection process.

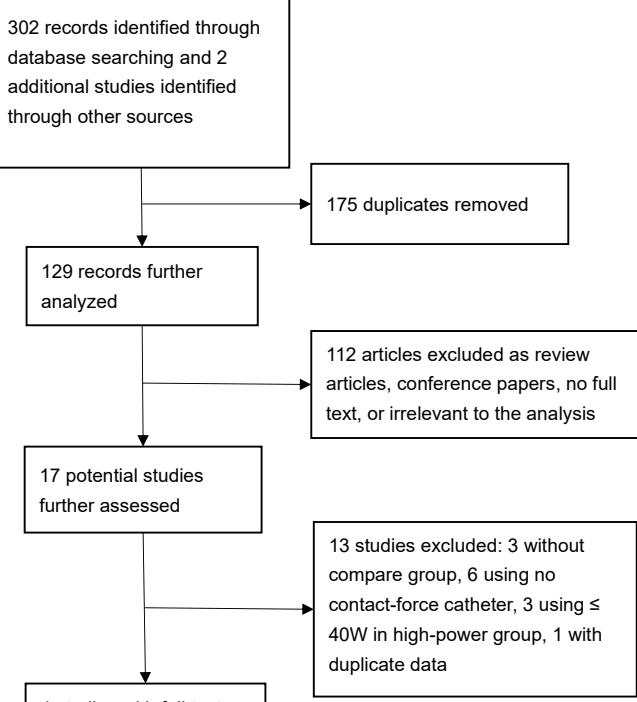

4 studies with full text

were selected in our

meta-analysis 
Figure2. Forest plot of the primary outcomes of selected studies comparing high power (HP) versus conventional power (CP) ablation of AF. Fig2A. Forest plot of AF recurrence; Fig2B. Forest plot of Complications.

A. Forest plot of AF recurrence

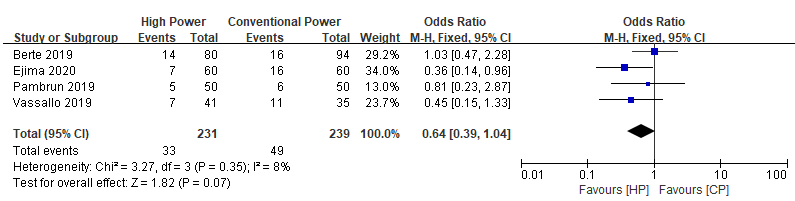

B. Forest plot of Complications

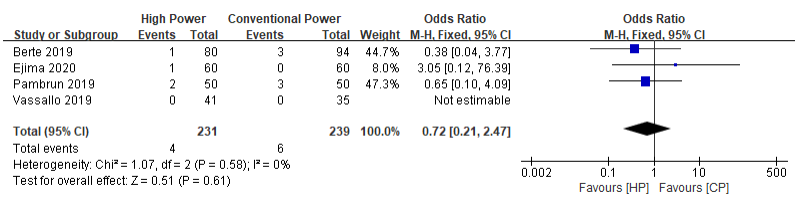


Figure3. Forest plot of the secondary outcome of selected studies comparing high power (HP) versus conventional power (CP) ablation of AF. Fig3A. Forest plot of acute reconnections of pulmonary veins; Fig3B. Forest plot of Ablation time; Fig3C. Forest plot of total procedure time.

A. Forest plot of acute reconnection of pulmonary veins

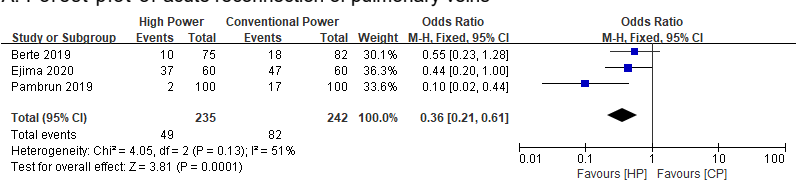

B. Forest plot of Ablation time

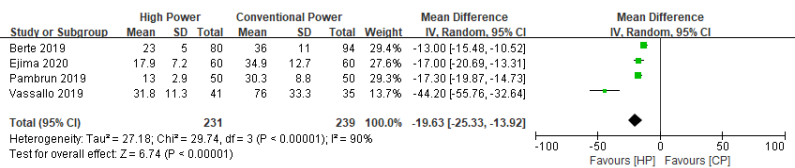

C. Forest plot of total procedure time

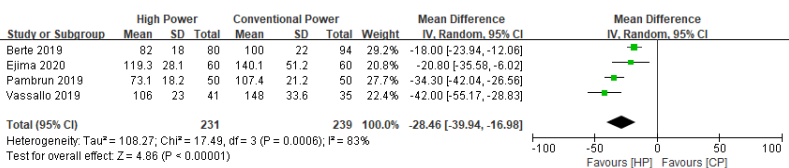


1 Figure4. Funnel plot of the selected studies in meta-analysis

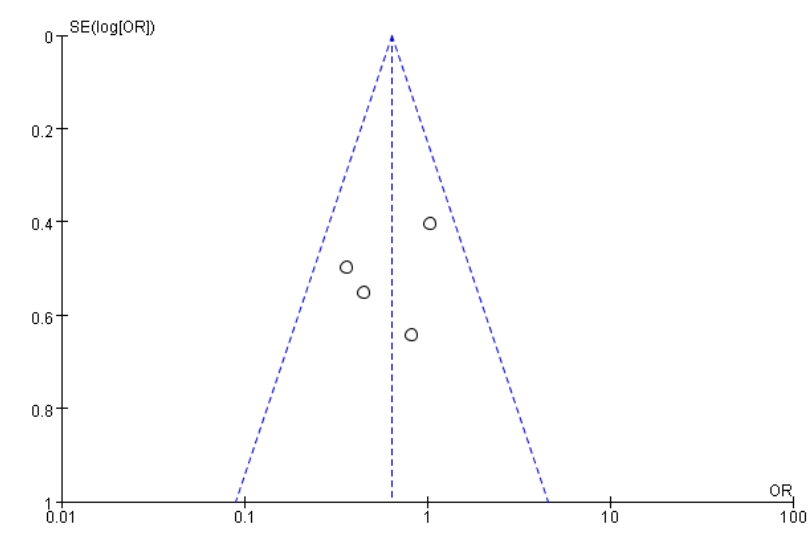

